\title{
Case Report: Mucinous Adenocarcinoma Arising From Congenital Ejaculatory Duct Cyst
}

\author{
Hua Shen ${ }^{1 *}$, Kai Liao ${ }^{1}$, Weili Wu ${ }^{1}$, Gongyu $\mathrm{Li}^{1}$, Shijin $\mathrm{Chen}^{2}$, Nan $\mathrm{Nan}^{3}$, Hongbo $\mathrm{Yu}^{1}$ and \\ Hongfei $W u^{1}$ \\ ${ }^{1}$ Department of Urology, BenQ Medical Center, The Affiliated BenQ Hospital of Nanjing Medical University, Nanjing, China, \\ 2Department of Pathology, BenQ Medical Center, The Affiliated BenQ Hospital of Nanjing Medical University, Nanjing, China, \\ ${ }^{3}$ Department of Radiology, BenQ Medical Center, The Affiliated BenQ Hospital of Nanjing Medical University, Nanjing, China
}

OPEN ACCESS

Edited by:

József Tímár.

Semmelweis University, Hungary

\section{${ }^{*}$ Correspondence:} Hua Shen shnjmu@hotmail.com

Received: 10 July 2020 Accepted: 05January 2021 Published: 26 March 2021

Citation:

Shen H, Liao K, Wu W, Li G, Chen S, Nan N, Yu H and Wu H (2021) Case Report: Mucinous Adenocarcinoma Arising From Congenital Ejaculatory

Duct Cyst.

Pathol. Oncol. Res. 27:528050.

doi: $10.3389 /$ pore. 2021.528050
Herein we present a previously unreported rare case of mucinous adenocarcinoma arising from a congenital ejaculatory duct cyst. Radiographic and endoscopic examinations revealed the tumor occurred in a cyst running through the prostate. Initially, the immunohistochemical pathology results showed that it was a metastatic mucinous adenocarcinoma, but no other primary lesions were clinically evidenced. Based on the embryonic development process of the male urogenital tract, the malformation of the patient's ejaculatory duct, and the pathological examination of the resected specimen, we considered the tumor to be a primary mucinous adenocarcinoma which originating from the hypoplastic ejaculatory duct. The tumor may have developed from the foci of intestinal metaplasia from cloacal remnants during embryonic development.

Keywords: mucinous adenocarcinoma, ejaculatory duct, ejaculatory duct cyst, ejaculatory duct tumor, ejaculatory duct adenocarcinoma

\section{INTRODUCTION}

The ejaculatory tube is composed of the excretory tube of the seminal vesicle and the vas deferens. Bilateral tubes enter into the base of the prostate and opening on both sides of the membrane of verumontanum. Ejaculatory duct cyst is rare congenital or acquired lesion, with fewer than 30 previous reports in the literature [12]. Only two cases of ejaculatory duct tumors have been reported, including one case of adenomatoid tumor of the ejaculatory duct [2] and another case of intraductal carcinoma of the prostate in the ejaculatory duct [9].

Mucinous adenocarcinomas are malignant tumor which usually occur in the gastrointestinal tract and are rare in the genitourinary tract. We report a case of primary mucinous adenocarcinoma arising from a congenital ejaculatory duct cyst, which may have been related to the cloacal remnants during embryonic development.

\section{CASE REPORT}

A 74-year-old man who was married with a son and daughter was admitted because of intermittent mucoid discharge from his urethra and difficulty voiding for 3 years. Indwelling catheterization was performed because of urinary retention. Digital rectal exanmination revealed a suspicious soft cystic mass with an unclear boundary in the upper left area of the moderately enlarged prostate. Serum prostate-specific antigen (PSA) was $4.12 \mathrm{ng} / \mathrm{ml}$. Imaging studies (Figure 1) 

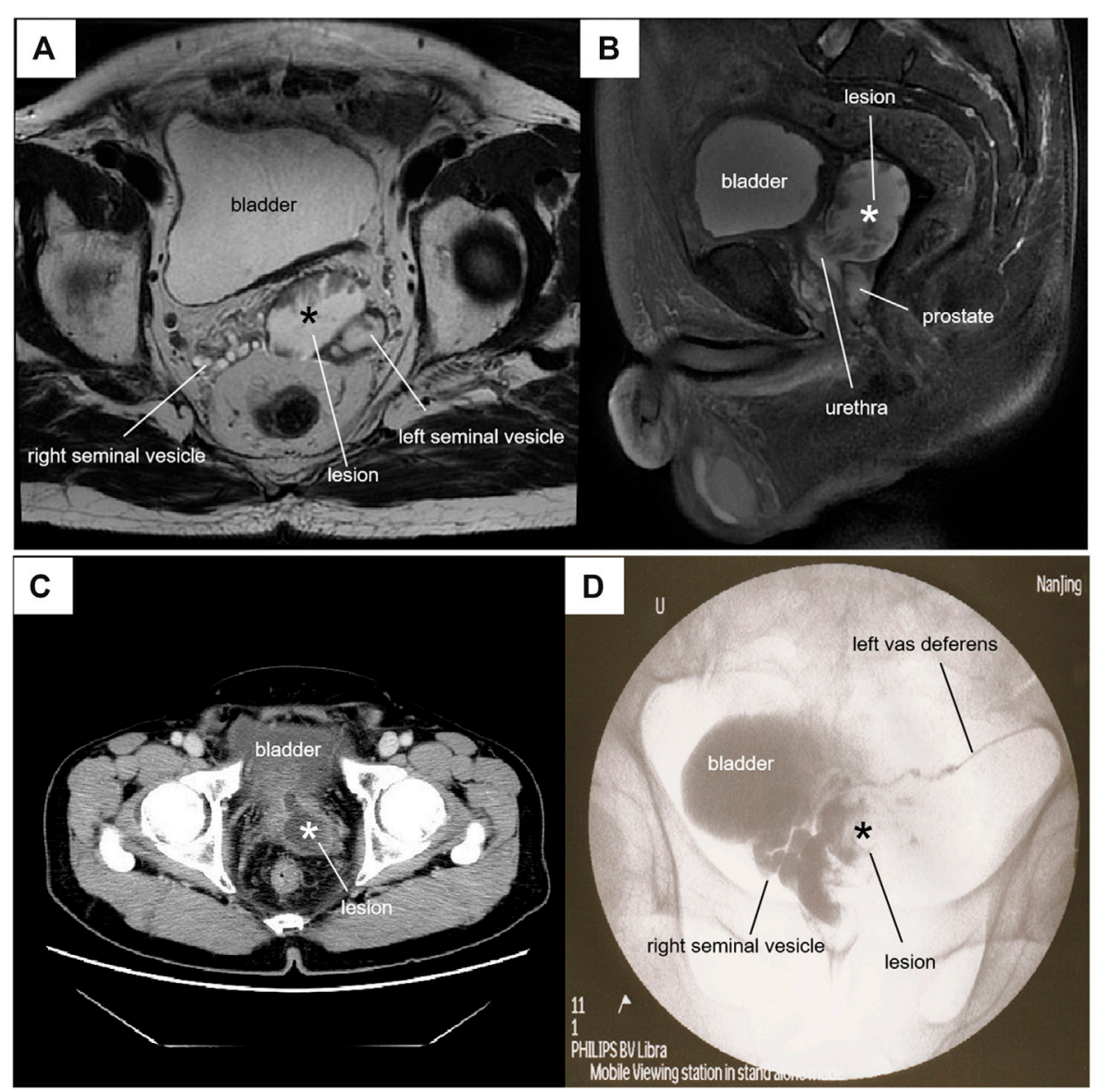

FIGURE 1 | Radiographic features of the lesion*. (A) Axial T2-weighted MRI showed a $45 \times 36 \times 44-m m$ lesion with mixed signals at the left lower posterior of the bladder. (B) Sagittal fat-suppressed T2-weighted MRI showed that the cystic-solid lesion passed through the prostate into the posterior urethra. (C) Contrast-CT showed a cystic-solid mass with obvious enhancement of the cyst wall communicating with the urethra. (D) Vasography showed a filling defectan in the cystic structure.

and an endoscopic examination (Figure 2) were subsequently performed. Magnetic resonance imaging (MRI) detected an irregular cystic-solid mass with villiform long $\mathrm{T} 1$ and $\mathrm{T} 2$ signals in the left ejaculatory duct area. Vasography showed an incomplete cyst-like structure with a filling defect under the bladder. Cystoscopy disclosed a cystic cavity filled with mucoid substance and multiple papillary neoplasms, which opened into the prostatic urethra near the top of the verumontanum. The cystoscope could be inserted into the right seminal vesicle through the urethral opening. Biopsies showed that the neoplasm was a metastatic mucinous adenocarcinoma or tubulo-villous adenoma. Immunohistochemistry revealed it to be a mucinous adenocarcinoma. All gastrointestinal tract tumor markers were normal. Computed tomography (CT) scanning showed a cysticsolid mass occupying the left ejaculatory duct area without renal agenesis. Contrast agent revealed an obviously enhanced cyst wall and a papillary lesion at the junction of the sigmoid colon and rectum. Colonoscopy revealed a $2.0-\mathrm{cm}$ polypoid mass in the proximal rectum. An endoscopic polypectomy was performed and the pathological result was a tubulo-villous adenoma. A radical prostatectomy and seminal vesiculectomy were then performed. The pathological diagnosis was adenocarcinoma with 55\% mucinous adenocarcinoma (enteric-type). No lipochrome pigment granules were oberved in the tumor. Immunohistochemistry showed PSA (-), P504S (+), 34ßE12 $(+/-)$, P63 (-), ERG (-), CK7 (+), CK20 (+), CDX-2 (3+), SATB-2 (-), CEA $(-)$, AR $(-)$, Ki-67 $(40 \%+)$, PAX2 (-), PAX8 $(-)$ and MUC6 $(2 \%+)$ (Figure 3).

\section{DISCUSSION}

Mucinous adenocarcinomas are a special pathological type of adenocarcinoma, in which $>50 \%$ of the lesion is composed of pools of extracellular mucin [3]. Of all colorectal cancers, $5-15 \%$ meet the criteria for mucinous adenocarcinoma [4]. Mucinous adenocarcinomas of the prostate, in which $>25 \%$ of the lesion is extravasated mucin, are one of the rarest morphological variants of all prostatic carcinomas, with an incidence of $0.21-0.43 \%$ [1]. The more common metastatic mucinous adenocarcinoma of the prostate is often secondary to colorectal, bladder or urethral tumors [8]. Mucinous 


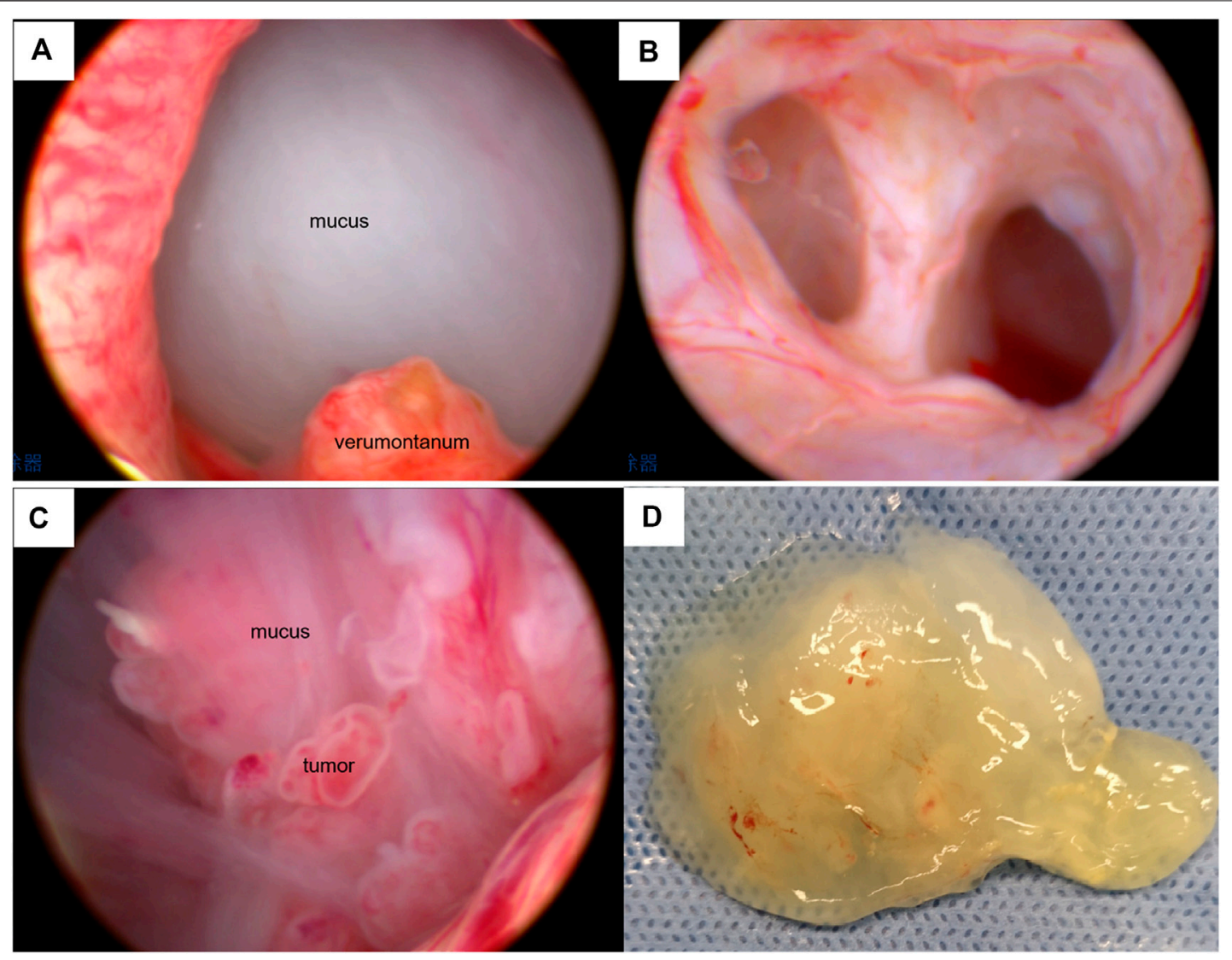

FIGURE 2 | Cystoscopic features of the lesion. (A) Spherical mucus-like barrier in the prostatic urethra under cystoscopy. (B) The cystoscope entered the right seminal vesicle. (C) Cystoscopy showed a mucoid substance and multiple papillary neoplasms in the cystic cavity. (D) Jelly-like substance sucked out from the cystic cavity.

adenocarcinomas of the ejaculatory duct have not previously been reported.

This case was pathologically confirmed as a mucinous adenocarcinoma, occurring in a cystic structure with invasion of the left seminal vesicle and vas deferens. Three-dimensional radiological reconstruction and histological examination revealed no normal ejaculatory duct was found in the left prostate lobe. The cyst, which was located at the left lower posterior of the bladder, passed through the prostate and connected to the right seminal vesicle through the prostatic urethra (Figure 1). We therefore considered this cystic structure to be a dysplastic left ejaculatory tube. Negative PSA, CEA and AR expressions excluded primary prostate or seminal vesicle adenocarcinoma. Although the tumor could be detected in the left seminal vesicle, and P504S was positively expressed in the seminal vesicles and vas deferens, the primary seminal vesicle carcinoma showed mostly CK7+ and CK20- immunostaining [7]. In this case, the tumor was $\mathrm{CK}_{20} 0^{+}, \mathrm{PAX}^{-}$and $\mathrm{PAX}^{-}$, indicating that it was not derived from the mesonephric or paramesonephric duct. Thus, the tumor likely did not arise from the seminal vesicle or vas deferens. Strong $\mathrm{CDX}-2^{+}$expression indicated that it was either metastatic colorectal mucinous adenocarcinoma or intestinal metaplasia, but no clinical evidence was found to support colorectal tumor metastasis, other than a rectal tubulo-villous adenoma.

The cloaca is the common compartment in the urogenital and anorectal channels in the 5-week-old human embryos. During weeks six to seven of embryonic development, the urorectal septum subdivides the cloaca into two separate parts [5]. The allantoic canal in the upper part of the primitive urogenital sinus forms the bladder and urethra, while the prostate develops from the lower urogenital sinus. The mesonephric duct is included in the vesicourethral canal within the urogenital sinus. Between weeks 12 and 13 of fetal development, the mesonephric duct develops and forms the epididymal duct, vas deferens and seminal vesicle under the action of testosterone. The ampulla of the vas deferens and seminal vesicle join posteriorly and superiorly to the prostate to form the ejaculatory duct, which opens into the prostatic urethra (Figure 4) [13]. In this case, the cystic ejaculatory duct developed a mucinous adenocarcinoma, which should have occurred in the colorectum. Although the tumor's precise pathogenesis was unknown, it may have developed from the foci of intestinal metaplasia from cloacal remnants, 

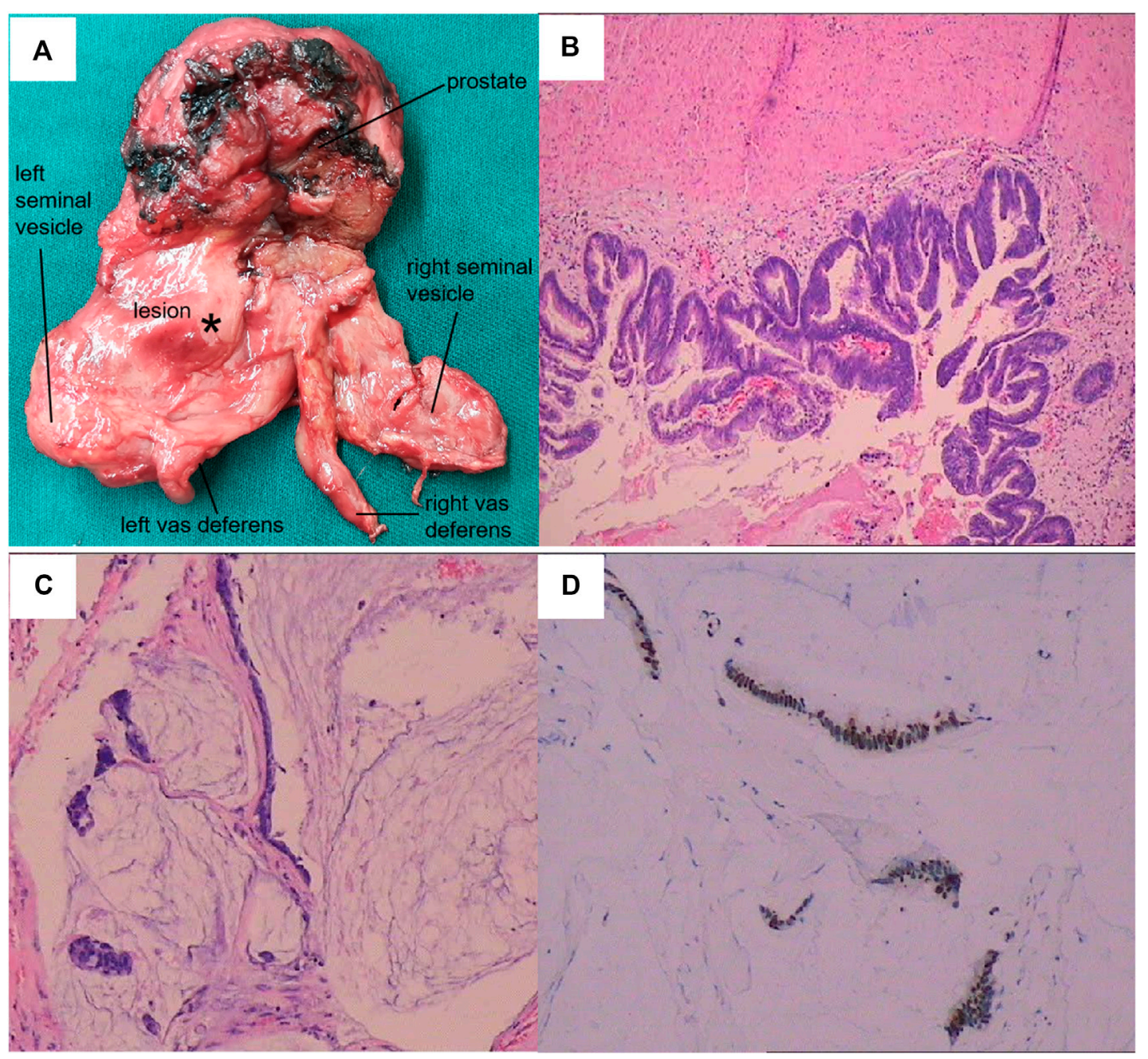

FIGURE 3 | Pathological features of the lesion. (A) General view of the resected specimen. (B) Hematoxylin and eosin (HE) staining of the neoplasm (100x). (C) HE staining of the mucinous area (100x). (D) Immunohistochemistry showed strong CDX-2 $2^{+}$expression.
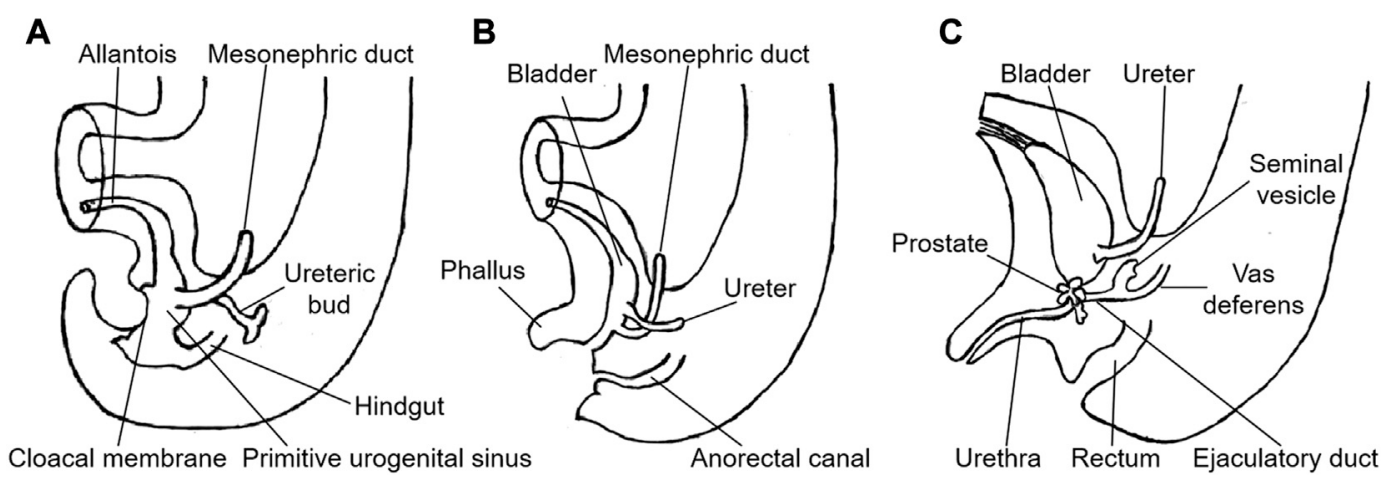

FIGURE 4 | Embryonic development of the male urogenital tract. (A) fifth week. (B) eighth week. (C) 13th week. (Redrawn from Langman's Medical Embryology. 13th Ed. TW Sadler. 2015: 259). 
which is similar to the genesis of primary vaginal adenocarcinoma with mucinous-enteric differentiation [14].

Interestingly, the initial pathological diagnosis suggested that the neoplasm in the cyst was a tubulo-villous adenoma, which also occurred in the patient's rectum. Of colorectal adenocarcinomas, 95\% develop from adenomas, which are classified as villous, tubular or tubulo-villous. The latter two are highly correlated with malignancy (including mucinous adenocarcinoma) because they contain villous tissue [6]. Several reports have also described villous adenomas of the urinary tract in which glandular epithelial lesions arose in the urothelial-lined urinary tract $[10,11]$. Cloacal tissue may remain in the urinary tract, with the potential to give rise to glandular epithelial neoplasms. In this case, the tubulo-villous adenoma may have occurred in the ejaculatory duct cyst with cloacal remnants and developed into mucinous adenocarcinoma after stimulation by harmful substances in the urine retained in the cyst.

The patient underwent radical prostatectomy and seminal vesiculectomy. MRI performed 6 months postoperation showed normal signaling of the vesicourethral anastomosis area and no enlarged lymph node was detected in the pelvis. However, 15 months postoperation, the patient experienced difficulty urinating. Cystoscopic examination revealed neoplasms at the vesicourethral anastomosis. The sample obtained via transurethral resection was pathologically confirmed to be mucinous adenocarcinoma. Tumor-cell seeding through the mucus secreted by the tumor might have been the main cause of recurrence. Chemotherapy was recommended.

\section{REFERENCES}

1. Bohman, K. D., and Osunkoya, A. O. (2012). Mucin-producing tumors and tumor-like lesions involving the prostate: a comprehensive review. Adv. Anat. Pathol. 19 (6), 374-387. doi:10.1097/PAP.0b013e318271a361

2. Fan, K., and Johnson, D. F. (1985). Adenomatoid tumor of ejaculatory duct. Urology 25, 653-654. doi:10.1016/0090-4295(85)90307-3

3. Hamilton, S. R., and Aaltonen, L. A. (2000). World health organization classification of tumours (Lyon, France: IARC Press).

4. Kang, H., O'Connell, J. B., Maggard, M. A., Sack, J., and Ko, C. Y. (2005). A 10year outcomes evaluation of mucinous and signet-ring cell carcinoma of the colon and rectum. Dis. Colon Rectum 48, 1161. doi:10.1007/s10350-004-0932-1

5. Kruepunga, N., Hikspoors, J. P. J. M., Mekonen, H. K., Mommen, G. M. C., Meemon, K., Weerachatyanukul, W., et al. (2018). The development of the cloaca in the human embryo. J. Anat. 233 (6), 724-739. doi:10.1111/joa. 12882

6. Morson, B. C., and Sobin, L. H. (1976). "Histological typing of intestinal tumours," in International histological classification of tumours. No. 15. Geneva: World Health Organization, 69.

7. Ormsby, A. H., Haskell, R., Jones, D., and Goldblum, J. R. (2000). Primary seminal vesicle carcinoma: an immunohistochemical analysis of four cases. Mod. Pathol. 13 (1), 46-51. doi:10.1038/modpathol.3880008

8. Osunkoya, A. O., Nielsen, M. E., and Epstein, J. I. (2008). Prognosis of mucinous adenocarcinoma of the prostate treated by radical prostatectomy: a study of 47 cases. Am. J. Surg. Pathol. 32 (3), 468-472. doi:10.1097/PAS. 0b013e3181589f72

\section{ETHICS STATEMENT}

Written informed consent was obtained from the individual(s) for the publication of any potentially identifiable images or data included in this article.

\section{AUTHOR CONTRIBUTIONS}

HS collected and analyzed the patient's clinical data, performed the review of literature and drafted the manuscript. KL, WW and GL participated in the collection and editing of the pictures. SC helped interpret pathological images and NN helped interpret the radiological images. HY and HW contributed to reviewing and revising the discussion. All authors have read and approved the final version of the manuscript.

\section{FUNDING}

Supported by Nanjing Medical Science and Technique Development Foundation (QRX17099).

\section{CONFLICT OF INTEREST}

The authors declare that the research was conducted in the absence of any commercial or financial relationships that could be construed as a potential conflict of interest.

9. Sanchez-Salazar, A. J., Basler, J. W., and Nicolas, M. M. (2010). Intraductal carcinoma of the prostate in the ejaculatory duct. Int. J. Surg. Pathol. 18, 298-299. doi:10.1177/1066896910364534

10. Seibel, J. L., Prasad, S., Weiss, R. E., Bancila, E., and Epstein, J. I. (2002). Villous adenoma of the urinary tract: a lesion frequently associated with malignancy. Hum. Pathol. 33 (2), 236-241. doi:10.1053/hupa.2002.31293

11. Wang, J., and Manucha, V. (2016). Villous adenoma of the urinary bladder: a brief review of the literature. Arch. Pathol. Lab Med. 140 (1), 91-93. doi:10. 5858/arpa.2014-0198-RS

12 Wang, S., Chen, S. W., Liu, W., Zhu, H. J., Jiang, H., Wang, Y. B., et al. (2007). Laparoscopic excision of extraprostatic ejaculatory duct cyst. Andrologia 39, 81-86. doi:10.1111/j.1439-0272.2007.00768.x

13. Wein, A. J. (2012). Campbell walsh-urology. 10th Edn. Philadelphia, PA: Saunders Elsevier, 1012-1013.

14. Werner, D., Wilkinson, E. J., Ripley, D.and Yachnis, A. (2004). Primary adenocarcinoma of the vagina withmucinous-entericdifferentiation: a report of two cases with associated vaginal adenosis without history of diethylstilbestrol exposure. J. Low. Genit. Tract Dis. 8 (1), 38-42. doi:10. 1097/00128360-200401000-00009

Copyright $\odot 2021$ Shen, Liao, Wu, Li, Chen, Nan, Yu and Wu. This is an open-access article distributed under the terms of the Creative Commons Attribution License (CC $B Y)$. The use, distribution or reproduction in other forums is permitted, provided the original author(s) and the copyright owner(s) are credited and that the original publication in this journal is cited, in accordance with accepted academic practice. No use, distribution or reproduction is permitted which does not comply with these terms. 